\title{
POSTASPHYXIAL HYPOXIC-ISCHEMIC ENCEPHALOPATHY
}

Models of association between risk factors and severe adverse outcome within 4 hours of birth were determined in 178 infants with postasphyxial hypoxic-ischemic encephalopathy (PA-HIE) admitted consecutively between 1985 and 1992 to the regional referral neonatal ICU at the Hospital for Sick Children, Toronto, Canada. Of 165 infants with known outcomes, 88 (53\%) had severe adverse outcome, 48 died (42 in the first month), and 40 had severe impairment and microcephaly at 1 year. Rates of PA-HIE and severe adverse outcome were 0.4 and $0.2 / 1000$ births; the rate of PA-cerebral palsy was $0.1 / 1000$ births. Delayed onset of breathing, administration of chest compressions, and seizures at less than 4 hours of age were most predictive of adverse outcome. (Ekert P, Perlman M, Steinlin M, Hao Y. Predicting the outcome of postasphyxial hypoxic-ischemic encephalopathy within 4 hours of birth. I Pediatr Oct 1997;131:613-617). (Reprints: Max Perlman MB, FRCP, Division of Neonatology, Department of Pediatrics, Hospital for Sick Children, 555 University Aver, Toronto, Ontario, M5G 1X8, Canada).

COMMENT. Three independent predictors in the first 4 hours after birth may be used as clinical markers of severe adverse outcome of asphyxia and HIE: apnea duration, chest compressions, age at onset of seizures. Measurements of $\mathrm{CBF}$ with PET during the neonatal period may also be predictive of childhood neurologic outcome.

Neonatal cerebral blood flow (CBF) and childhood IQ and neurologic outcome. A significant negative correlation between neonatal $\mathrm{CBF}$ and childhood IQ measured at ages 4 to 12 years was found in a study at Washington University School of Medicine, St Louis, MO. The mean neonatal $\mathrm{CBF}$ in 8 with abnormal childhood neurologic outcome was higher than in 8 with normal neurologic evaluations. Higher CBF correlated with lower IQ. (Rosenbaum JL, Almli CR, Yundt KD, Altman DI, Powers WJ. Higher neonatal cerebral blood flow correlates with worse childhood neurologic outcome. Neurology Oct 1997;49:1035-1041).

\section{CONGENITAL PORENCEPHALY AND HIPPOCAMPAL SCLEROSIS}

MRI volumetric findings were correlated with seizure patterns and EEGs in 14 patients with intractable seizures, porencephaly and hippocampal sclerosis (HS) in a study at the University of Alabama, Birmingham, AL. Psychoparetic complex partial seizures (CPS) occurred in 10, simple partial seizures in 3, and generalized TC seizures in 1. EEGs showed ictal or interictal temporal localization in $9(64 \%)$ patients with CPS. Porencephaly was distant from the temporal area and in the middle cerebral artery distribution in 8 ; it was related to the posterior cerebral in only 3 . Hippocampal formation atrophy in $13(93 \%)$ patients was concordant with CPS and EEG temporal localization in $70 \%$ cases. Ten had amygdala atrophy, concurrent with HS in $57 \%$. Two with HS were seizure free after temporal lobectomy. A common ischemic pathogenesis is proposed for the dual pathology involving both porencephaly and mesial temporal sclerosis. HS is the most likely origin for CPS in patients with EEG temporal localization. (Ho SS, Kuzniecky RI, Gilliam F, Faught E, Bebin M, Morawetz R. Congenital porencephaly and hippocampal sclerosis. Clinical features and epileptic spectrum. Neurology Nov 1997;49:1382-1388). (Reprints: Dr Ruben I Kuzniecky, Department of Neurology, UAB Station, Birmingham, AL 35294).

COMMENT. Patients presenting with intractable complex partial seizures 
and congenital porencephaly should be evaluated with MRI for coexistent mesial temporal sclerosis. Hippocampal formation atrophy is the more likely origin for the seizures in patients with dual pathologies, particularly when the EEG shows localization to the temporal lobe.

Quantitative MRI of the hippocampus (Van Paesschen W et al. Ann Neurol Nov 1997;42:756-766), and Proton magnetic resonance spectroscopic imaging (Cendes F et al. Ann Neurol Nov 1997;42:737-746) were used in the presurgical evaluation of temporal lobe epilepsy at the National Hospital, London, UK, and Montreal Neurological Institute, Canada.

\section{CEREBELLAR DISORDERS}

\section{CEREBELLAR STRUCTURAL ABNORMALITIES AND GENETICS}

The etiology and incidence of known metabolic and hereditary disorders associated with unilateral or bilateral structural cerebellar abnormalities, defined by CT and/or MRI, were determined in 78 children examined at the University Hospital Aachen, Germany, and Katholieke Universiteit Leuven, Belgium. Lesions were bilateral in 62 and unilateral in 16, both cerebellar hemispheres were involved in 38 , the vermis in 15 , and pontocerebellar in 9 . Hemisphere atrophy was static in 10 and progressive in 28. MRI was superior to CT in definition of lesions. Genetic/metabolic causes were found in more than half the cases of ponto-cerebellar hypoplasia or progressive cerebellar atrophy, but in none with unilateral cerebellar lesions. These included amino and organic acidurias, lactic acidosis, lysosomal and peroxisomal disorders, Menkes kinky hair disease, molybdenum cofactor deficiency, and autosomal dominant ataxias. Other causes of cerebellar and pontocerebellar hypoplasia included intrauterine ionizing radiation, phenytoin exposure, cytomegalovirus, chromosomal syndromes, hypogonadism, Ito's hypomelanosis, and carbohydrate-deficient-glycoproteins syndromes. Investigations should include EEG, EMG and NCS, abdominal ultrasound, urine screening for amino and organic acids, and blood tests for acanthocytes, liver function, protein electrophoresis, ammonia, lactate and pyruvate, copper and ceruloplasmin, immunoglobulins, VLC fatty acids, and glycoproteins. An overview of the literature is also presented. (Ramaekers VTh, Heimann G, Reul J, Thron A, Jaeken J. Genetic disorders and cerebellar structural abnormalities in childhood. Brain Oct 1997;120:1739-1751). (Respond: Dr V Th Ramaekers MD, Department of Paediatrics, Medizinische Einrichtungen der RWTH, Pauwelsstrasse 30, D52057 Aachen, Germany).

COMMENT. Pontocerebellar hypoplasia or progressive cerebellar atrophy defined by MRI is an indication for biochemical and neurophsiological tests for hereditary or degenerative neurological disorders.

Focal cerebellar lesions and associated learning impairments were detected in 8 patients cf 6 control subjects tested in a serial reaction-time task at the Catholic University, and University of Rome 'La Sapienza', Rome, Italy. (Molinari M et al. Brain Oct 1997;120:1753-1762). Cerebellar patients had longer reaction times than controls when stimuli were presented in sequence.

\section{DEMYELINATING DISORDERS}

\section{OPTIC NEURITIS AND RISK OF MULTIPLE SCLEROSIS}

Risk factors for the development of multiple sclerosis (MS) were 